following results were obtained:

1) The primary positive potential is not clear in association area, and is clear in sensory and motor area.

2) Infant cortex does not show these differentiation of cortical response.

3) In the case of brain tumor which was situated just under the surface of the cortex, the RR was not obtained in the cortex over the region involved by the tumor.

\title{
135. Studies on the Convulsive Seizure induced by $\gamma$-Guanidinobutyric Acid
}

\author{
Aijiro Sawal, Naoji Takeuchi, Akitane Mori and Dennosuke Jinnal \\ Dept. of Neurosurgery, Osaka Univ. Medical School
}

$\gamma$-Guanidinobutyric acid ( $\gamma$-GBA) has already been shown to exist in human brain and other vertebrate tissues by Irreverre et al. According to the report, human brain contains $5-10 \mu \mathrm{g} / \mathrm{g}$ wet weight of it. As to the physiological investigation of $\gamma$-GBA, it has been shown to have an inhibitory effect on the crayfish stretch receptor like $\gamma$-aminobutyric acid (GABA). However, no physiological studies have as yet been carried out with mammalian systems. Pisano et al have reported that $\gamma$-GBA is synthesized in mammals by a transamidination reaction involving GABA and an amidine donor such as arginine. This suggests that the reaction requires 2 moles of ammonia, and it is very important to study the increasing process of ammonia during convulsive seizure. Our present reports show the results of physiological and biochemical studies on the convulsive seizure induced by the intracisternally administered $\gamma-\mathrm{GBA}$ and the effects of several electrolytes and vitamins on it.

Experimental results; When $\gamma$-GBA in dosage of $5 \mathrm{mg}$ per $\mathrm{kg}$ of body weight was injected into the cisterna magna of rabbit, clonic convulsion (KK) was repeatedly observed during about one hour. The latent period to produce $\mathrm{KK}$ was 14 to 15 minutes. We call the seizure $\gamma$-GBA convulsion.

Then effects of electrolytes and vitamins on $\gamma$-GBA convulsion were investigated. In case of intracisternal administration of calcium chloride $(3.2 \%, 0.5 \mathrm{ml})$, co-carboxylase $(200 \mu \mathrm{g})$, pyridoxal phosphate $(0.4 \mathrm{mg})$ and V. $\mathrm{B}_{12}(40 \mu \mathrm{g})$ individually with $\gamma$-GBA, no convulsive fit was observed. 